\title{
Pretreatment Patient Specific Quality Assurance and Gamma Index Variation Study in Gantry Dependent EPID Positions for IMRT Prostate Treatments
}

\author{
Siji Cyriac, ${ }^{1,2}$ M. M. Musthafa, ${ }^{3}$ R. Ganapathi Raman, ${ }^{1}$ K. Abdul Haneefa, ${ }^{4}$ and V. T. Hridya ${ }^{5}$ \\ ${ }^{1}$ Department of Physics, Noorul Islam Centre for Higher Education, Kumaracoil, Thuckalay, Kanyakumari District, \\ Tamil Nadu 629 180, India \\ ${ }^{2}$ Department of Radiation Therapy, HCG-Dr. Balabhai Nanavati Hospital, Vile Parle, Mumbai, Maharsahtra 400 056, India \\ ${ }^{3}$ Department of Physics, University of Calicut, Thenhipalam, Kerala 673635, India \\ ${ }^{4}$ INFN and Department of Physics, University of Turin, Via P. Giuria, 10125 Turin, Italy \\ ${ }^{5}$ Department of Radiation Therapy, K.S Hegde Medical Academy, Nitte University, Deralakatte, Mangalore, Karnataka 575018, India
}

Correspondence should be addressed to Siji Cyriac; sijicyriacsanju@gmail.com

Received 30 November 2013; Accepted 29 January 2014; Published 5 March 2014

Academic Editor: Tibor Major

Copyright (C) 2014 Siji Cyriac et al. This is an open access article distributed under the Creative Commons Attribution License, which permits unrestricted use, distribution, and reproduction in any medium, provided the original work is properly cited.

\begin{abstract}
Pretreatment quality assurance (QA) is a major concern in complex radiation therapy treatment plans like intensity modulated radiation therapy (IMRT). Present study considers the variations in gamma index for gantry dependent pretreatment verification and commonly practiced zero gantry angle verifications for ten prostate IMRT plans using two commercial medical linear accelerators (Varian 2300 CD, Varian Clinac iX). Two verification plans (the one with all fields at the actual treatment angles and one with all fields merged to 0 degree gantry angles) for all the patients were generated to obtain dose fluence mapping using amorphous silicon electronic portal imaging device (EPID). The gamma index was found depend on gantry angles but the difference between zero and the nonzero treatment angles is in the confidence level for clinical acceptance. The acceptance criteria of gamma method were always satisfied in both cases for two machines and are stable enough to execute the patient specific pretreatment quality assurance at 0 degree gantry angle for prostate IMRTs, where limited number of gantry angles are used.
\end{abstract}

\section{Introduction}

Modern cancer treatments using radiation therapy is mostly employed with multileaf optimized plans as in intensity modulated radiation therapy. Higher monitor units and continuous motion of multi-leafs during the beam on time need to be strictly monitored for beneficial outcome from IMRT treatments. Complex IMRT plans are widely used in routine clinical practice which requires pretreatment patient specific quality assurance tests $[1,2]$. Pretreatment quality assurance in complex treatment techniques like intensity modulated radiation therapy is carried out using electronic portal imaging devices attached to the medical linacs. This ensures the accuracy of treatment plan generated using commercial planning systems for complex IMRT treatments.
Pretreatment quality verification is advisable in all IMRT treatment plans to compare the beam fluence maps delivered using continuous motion of multileaf collimators (MLC). Most centers execute the patient specific verification plans generated prior to the first treatment fraction, where all the fields with various gantry angles are merged to zero degree gantry angle and dose fluence generated from TPS is cross-checked using EPID outputs [3, 4]. The linacs attached with EPID make the pretreatment QA easier and faster than other commercial tools like 2D detector arrays, which is time consuming for machinery settings. EPIDs provide high resolution two-dimensional data. It requires virtually no time for patient set-up or postprocessing to obtain useful data with a fast image acquisition which is stored in digital for further analysis [5-8]. 
Apart from usual zero degree gantry angles, many studies $[9,10]$ investigated the fluence matching in oblique angles. Gravitational sagging of linac head and EPID tail can influence the quality assurance $[11,12]$. However, if the sagging is minimal, we can achieve the actual treatment delivery positions for pretreatment verification purpose. This helps us to get the actual dose mapping in EPIDs. The MLC motion and collimator rotations can be influenced by the gravity shifts in nonzero gantry angles. This will be more severe in IMRT treatments where the critical organs are closely covered using MLCs.

This work aims to compare IMRT pretreatment verification for prostate plans, from two commercial medical linac accelerator machines where on-board EPID (PortalVision, Varian Medical Systems, Palo Alto, CA) is available. Dose fluence mapping were performed using EPIDs, to compare with the TPS generated dose maps using gamma index technique ( $3 \mathrm{~mm}, 3 \%$, integrated in Eclipse TPS) with two methods: one with actual treatment gantry angles and the other with all gantry angles merged to zero degree gantry angle.

\section{Materials and Methods}

Measurements were taken from two commercial medical linear accelerators-Varian Clinac 2300CD and Varian Clinac iX (Varian Associates, Palo Alto, CA). Clinac 2300 CD is equipped with 80 dynamic MLC and Clinac iX is equipped with Millennium 120 dynamic MLC. Both machines are fully commissioned for treating patients using IMRT technique. Both machines have 6 and $18 \mathrm{MV}$ photon beams and onboard amorphous-silicon EPID (aS500-Varian Medical Systems, Palo Alto, CA). EPID can be positioned at all gantry angles by motorized robotic, three-axis extract arms [13].

Ten prostate IMRT plans were generated for our study with gantry angles; $0^{\circ}$ (anterior), $75^{\circ}$ (right anterior oblique), $135^{\circ}$ (right posterior oblique), $225^{\circ}$ (left posterior oblique), and $285^{\circ}$ (left anterior oblique) using $6 \mathrm{MV}$ photon energies as shown in Figure 1. Varian Eclipse v.n.11.0 treatment planning system (Varian Medical Systems, Palo Alto, CA) was used to create the plans, where analytical anisotropic analytical algorithm (AAA) [14] is available for the optimization. Target and critical organ delineations were performed by the same radiation oncologist and the same medical physicist who did the dosimetric optimizations in all plans. Our linacs are precalibrated with farmer ionization chamber $\left(0.6 \mathrm{~cm}^{3}\right.$ PTW 30001) and associated PTW Freiburg electrometer (PTW 10008), using TRS 398 Dw protocol [15].

As per the Atomic Energy Regulatory Board of India (AERB) [16] safety rules, the leaf positions are periodically checked and documented for both machines. A mechanical front pointer made up of steel is attached to linac head to determine the distance between the target and the surface of the EPID. Pin point maker was drawn in "standard graph" and pasted on EPID using the front pointer while the gantry is at zero degree angle. The deviations from the marked position in all $(X, Y$, and $Z)$ directions are noted while the gantry is rotated. The measurements are noted for standard field size

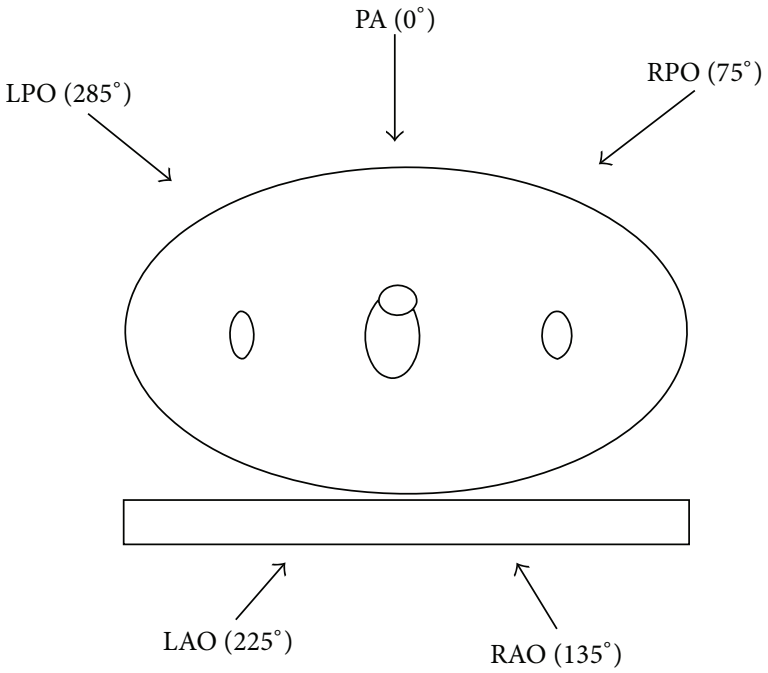

FIGURE 1: Gantry angle directions in 5-field prostate IMRT treatment.

of $10 \times 10 \mathrm{~cm}^{2}$ for $0^{\circ}, 60^{\circ}, 120^{\circ}, 180^{\circ}$, and $240^{\circ}$ gantry angles. These measurements will give the EPID and gantry deviation while gantry on rotation.

The MLC position checks are performed in different ways during the study. Standard shapes (say "E") are imported from TPS to linac and performed the reproducibility on EPID for various gantry angles. Monthly checks on MLC leaf position is to irradiate an imported (from TPS) image using radiographic films and is done on zero degree gantry angle. No significant sagging, dislocations, or misalignments in collimator leaf positions were noticed while rotation during this study. Leaf position accuracy is studied on both machines using radiographic films in limited gantry angles. The measurement result of radiographic film on zero degree gantry angle from Clinac $2300 \mathrm{CD}$ is shown in Figure 4, where alternate MLC leafs from both sides are closed to generate compatible shape for MLC position accuracy study, projecting to the isocenter. These studies give the confidence on the MLC position accuracy and are essential to make sure any misalignments of multileafs are under threshold for clinical acceptance.

Two sets of verification plans were created, one with the actual gantry angle and the other with all fields merged to zero degree gantry angle. Varian medical system has dedicated portal dosimetry software and is embedded in our Eclipse planning system [17]. This software can compare the dose fluence map using gamma index [18] method. The treatment plans generated from TPS were compared with the EPID measured dose maps. In the low gradient regions; the mean difference in dose should not be greater than $3 \%$ of prescribed dose. On the same time, in high gradient region the distance to agreement should not be greater than $3 \mathrm{~mm}$. This was the default and acceptable settings for all IMRT plans in our institute, which is followed in this study as well.

Gamma index pretreatment evaluations were performed for ten patients in two machines separately for actual treatment gantry angles and for the zero degree gantry angles. 


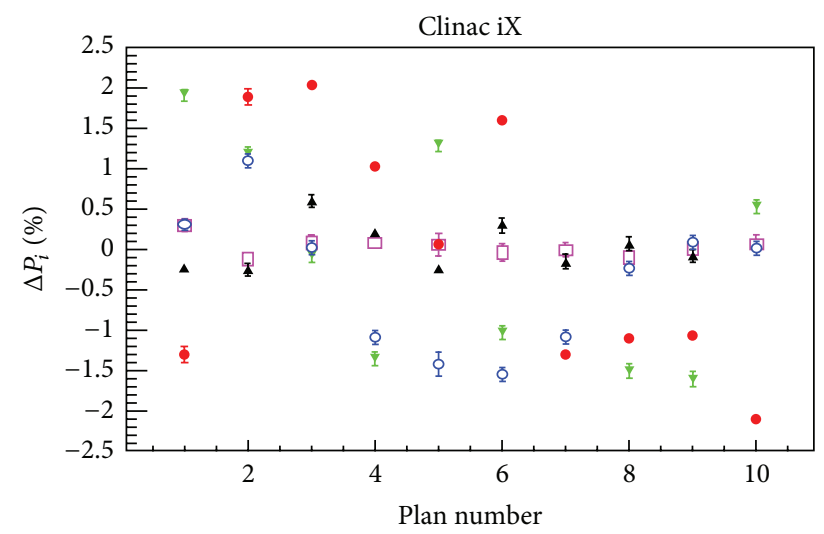

$\begin{array}{ll}\text { Gantry angle: } & \\ \square 0 & \checkmark 225 \\ \text { \ } 75 & \circ 285 \\ \text { - } 135 & \end{array}$

Figure 2: Difference in gamma index values $\left(\Delta P_{i}\right)$ between zero degree gantry angle obtained by merging all gantry angles and individual gantry angles, obtained from Clinac iX machine plotted for ten patients $\left(x\right.$-axis $=$ patient number, $y$-axis $\left.=\Delta P_{i}\right)$.

The criterion of acceptability of the gamma evaluation requires that no more than $5 \%$ of the points should have a gamma value larger than one. The percentage of points satisfying the above passing criteria was found by comparing the TPS generated 2D gamma map with EPID provided $2 \mathrm{D}$ gamma map in zero and at actual gantry angles. The percentage difference in acceptance criteria for each field ( $i$ th) was calculated by subtracting the percentage obtained with actual gantry angles $\left(G_{\text {non_}_{0} i}\right)$ from that obtained with zero degree gantry angle $\left(G_{0 i}\right)$. ROOT v5-34 [19] software was used to plot these values after dependent Student's $t$-test for statistical acceptance $(P<0.05)$.

\section{Results and Discussion}

EPID shifts were found in daily quality assurance checks, for $10 \times 10 \mathrm{~cm}^{2}$ open square field projections at $0^{\circ}, 60^{\circ}, 120^{\circ}, 180^{\circ}$, and $240^{\circ}$ gantry angle positions. Maximum deviations in $X$, $Y$, and $Z$ shifts range from $-1.2 \mathrm{~mm}$ to $0.95 \mathrm{~mm},-0.75 \mathrm{~mm}$ to $0.68 \mathrm{~mm}$, and $-0.2 \mathrm{~mm}$ to $0.3 \mathrm{~mm}$, respectively. All the pretreatment quality assurance was carried out after checking the EPID shifts. The shifts in all directions are taken into account and corrected for all fields.

Gamma index acceptance criteria were always satisfactory in both linear accelerators for both zero degree gantry and nonzero gantry angle pretreatment quality assurance tests. The difference in two values $\left(\Delta P_{i}\right)$, one obtained with gantry at zero degree and the other with individualized gantry angles $\left(0^{\circ}, 75^{\circ}, 135^{\circ}, 225^{\circ}\right.$, and $\left.285^{\circ}\right)$ for all ten patients for Clinac iX and Clinac 2300 CD, are as shown in Figures 2 and 3 , respectively.

Both linacs have independent behavior in the gantry angle dependent pretreatment verifications. Maximum differences in gamma index values were obtained for Clinac iX

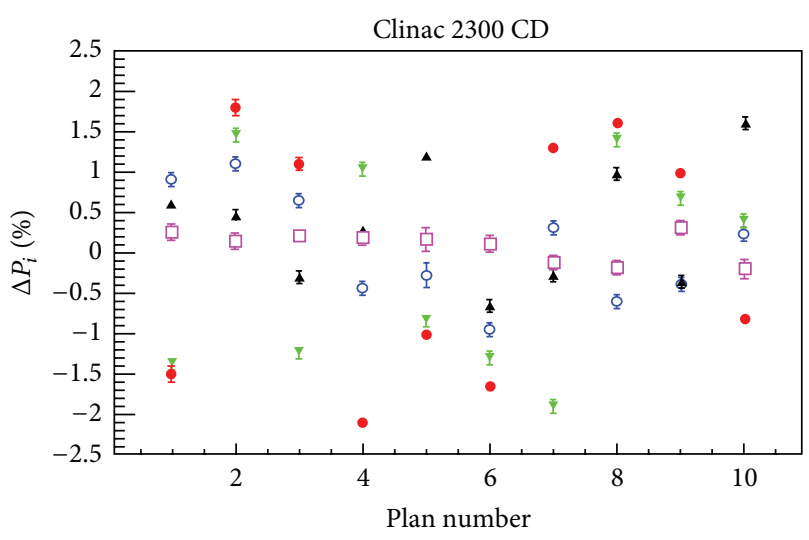

$$
\begin{aligned}
& \text { Gantry angle: } \\
& \square 0 \quad \text { v } 225 \\
& \text { ^ } 75 \quad 0285 \\
& \text { - } 135
\end{aligned}
$$

FIgURE 3: Difference in gamma index values $\left(\Delta P_{i}\right)$ between zero degree gantry angle obtained by merging all gantry angles and individual gantry angles, obtained from Clinac $2300 \mathrm{CD}$ machine plotted for ten patients $\left(x\right.$-axis $=$ patient number, $y$-axis $\left.=\Delta P_{i}\right)$.

machine. While for both the machines maximum difference in acceptance criteria was found in $135^{\circ}$ and $225^{\circ}$ gantry angles, the mean values of the percentage of the points having a gamma less than one for nonzero gantry angles in Clinac $2300 \mathrm{CD}$ machine are $0.353 \pm 0.024 \%\left(75^{\circ}\right),-0.0351 \pm 0.042 \%$ $\left(135^{\circ}\right),-0.168 \pm 0.03 \%\left(225^{\circ}\right)$, and $0.053 \pm 0.019 \%\left(285^{\circ}\right)$ and for Clinac iX machine are $0.0305 \pm 0.04 \%\left(75^{\circ}\right),-0.023 \pm 0.12 \%$ $\left(135^{\circ}\right),-0.172 \pm 0.43 \%\left(225^{\circ}\right)$, and $-0.155 \pm 0.29 \%\left(285^{\circ}\right)$. The mean value of the percentage that satisfies the gamma points passing criteria measured at zero degree for Clinac $2300 \mathrm{CD}$ and Clinac iX was $0.084 \pm 0.02(\%)$ and $0.037 \pm 0.014(\%)$, respectively. The student's $t$-test value were found to be 0.02 $(0.001<P>0.05)$ for all ten IMRT patients.

It is always advisable to check the gantry sagging effects for reasonable results from individualized gantry angle pretreatment quality assurance. This improves the IMRT quality and ensures the adequacy of the complex IMRT plans. Periodic mechanical quality assurance for gantry sagging using mechanical pointer attached to collimator must be carried out before IMRT treatments.

Besides the gantry sagging, MLC position accuracy must be studied independently to ensure the MLC position tolerance limits. During this study, collimator leaf position accuracy checks using light field on EPID surface for various gantry angles show negligible deviations. The zero degree gantry angle MLC position was checked for both the machines using EPIDs and radiographic films. Clinac iX and Clinac $2300 \mathrm{CD}$ had a little effect with a mean difference in various leaf positions ranging from about -0.075 to $0.06 \mathrm{~mm}$. The darkening of radiographic film always results in bigger uncertainties on these measurements. Figure 4 shows typical example for Clinac $2300 \mathrm{CD}$ leaf position accuracy study on EPID and radiographic film, where alternate MLC leafs are closed from both sides. 


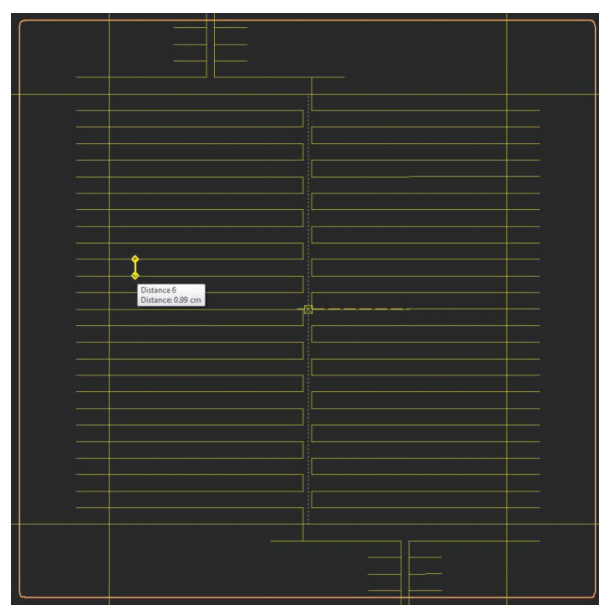

(a) TPS generated

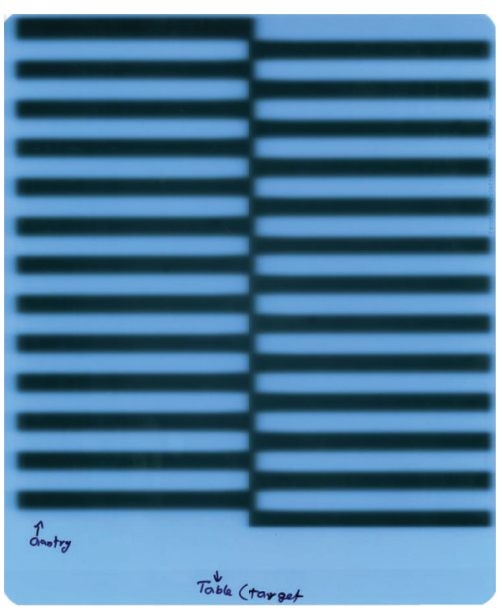

(b) Shape on radiographic film

FIGURE 4: MLC leaf position check from TPS (a) and verification using radiographic film (b) done at Clinac 2300 CD machine.

This study shows that the advantages of actual gantry pretreatment quality assurance are totally machine dependent. There is no significant difference obtained once the mechanical quality controls are performed for gantry sagging and MLC leaf positions besides other dosimetric checks. A definite number of gantry angle IMRT plans make these studies possible, while the gantry dependent quality assurance in more complex treatments and ARC therapies is practically time consuming and difficult in fluence mapping.

\section{Conclusion}

Patient specific pretreatment QAs should be better done at the treatment angles, if an on-board EPID is available. Pretreatment plan verifications using EPID are preferred to be in gantry angle dependent positions but it was also shown that such requirement is not strictly necessary. If the periodic quality assurances are performed, then the pretreatment IMRT QA could be executed at zero degree gantry angles as well, with considerable time reduction to perform the quality control for each patient. In fewer cases, the zero degree pretreatment analysis is even better than actual degree gantry angles. In a treatment facility where on-board EPID is not available, the time consuming gantry dependent QA using $2 \mathrm{D}$ arrays can be restricted to zero gantry angle pretreatment verifications. However, zero degree gantry angle pretreatment plan verifications cannot be employed in continuous arc treatments (i.e., gantry speed stability affects the dose delivery), where gantry dependent quality assurance is difficult.

\section{Conflict of Interests}

The authors declare that there is no conflict of interests regarding the publication of this paper.

\section{Acknowledgments}

The authors sincerely acknowledge the assistance provided by Mr. K. K. Shakir and Mr. A. Siddartha (Medical Physicists, AJ Cancer Institute, Mangalore, India) during the experiments. They are grateful to Professor Dr. Jayaram Shetty (Oncologist, K. S. Hegde Medical Academy, Mangalore, India) for his periodic suggestions.

\section{References}

[1] D. A. Low, S. Mutic, J. F. Dempsey et al., "Quantitative dosimetric verification of an IMRT planning and delivery system," Radiotherapy and Oncology, vol. 49, no. 3, pp. 305-316, 1998.

[2] S. C. Vieira, M. L. P. Dirkx, B. J. M. Heijmen, and H. C. J. de Boer, "SIFT: a method to verify the IMRT fluence delivered during patient treatment using an electronic portal imaging device," International Journal of Radiation Oncology Biology Physics, vol. 60, no. 3, pp. 981-993, 2004.

[3] M. van Zijtveld, M. Dirkx, M. Breuers, H. de Boer, and B. Heijmen, "Portal dose image prediction for in vivo treatment verification completely based on EPID measurements," Medical Physics, vol. 36, no. 3, pp. 946-952, 2009.

[4] A. S. Ali, M. L. P. Dirkx, R. M. Cools, and B. J. M. Heijmen, "Accurate IMRT fluence verification for prostate cancer patients using "in-vivo" measured EPID images and in room acquired kilovoltage cone-beam CT scans," Radiation Oncology, vol. 8, pp. 211-218, 2013.

[5] P. E. Engström, P. Haraldsson, T. Landberg, H. S. Hansen, S. A. Engelholm, and H. Nyström, "In vivo dose verification of IMRT treated head and neck cancer patients," Acta Oncologica, vol. 44, no. 6, pp. 572-578, 2005.

[6] P. B. Greer and C. C. Popescu, "Dosimetric properties of an amorphous silicon electronic portal imaging device for verification of dynamic intensity modulated radiation therapy," Medical Physics, vol. 30, no. 7, pp. 1618-1627, 2003.

[7] M. van Zijtveld, M. L. P. Dirkx, H. C. J. de Boer, and B. J. M. Heijmen, "Dosimetric pre-treatment verification of IMRT using 
an EPID; clinical experience," Radiotherapy and Oncology, vol. 81, no. 2, pp. 168-175, 2006.

[8] L. N. McDermott, M. Wendling, B. van Asselen et al., "Clinical experience with EPID dosimetry for prostate IMRT pretreatment dose verification," Medical Physics, vol. 33, no. 10, pp. 3921-3930, 2006.

[9] P. W. Chin, D. G. Lewis, and E. Spezi, "Correction for doseresponse variations in a scanning liquid ion chamber EPID as a function of linac gantry angle," Physics in Medicine and Biology, vol. 49, no. 8, pp. N93-N103, 2004.

[10] G. Yan, C. Liu, T. A. Simon, L.-C. Peng, C. Fox, and J. G. Li, “On the sensitivity of patient-specific IMRT QA to MLC positioning errors," Journal of Applied Clinical Medical Physics, vol. 10, no. 1, pp. 120-128, 2009.

[11] M. Mohammadi, E. Bezak, and P. Reich, "Verification of dose delivery for a prostate sIMRT treatment using a SLIC-EPID," Applied Radiation and Isotopes, vol. 66, no. 12, pp. 1930-1938, 2008.

[12] M. F. Clarke and G. J. Budgell, "Use of an amorphous silicon EPID for measuring MLC calibration at varying gantry angle," Physics in Medicine and Biology, vol. 53, no. 2, pp. 473-485, 2008.

[13] A. van Esch, T. Depuydt, and D. P. Huyskens, "The use of an aSi-based EPID for routine absolute dosimetric pretreatment verification of dynamic IMRT fields," Radiotherapy and Oncology, vol. 71, no. 2, pp. 223-234, 2004.

[14] W. Ulmer, J. Pyyry, and W. Kaissl, "A 3D photon superposition/convolution algorithm and its foundation on results of Monte Carlo calculations," Physics in Medicine and Biology, vol. 50, no. 8, pp. 1767-1790, 2005.

[15] International Atomic Energy Agency (IAEA), “Absorbed dose determination in external beam radiotherapy: an international code of practice for dosimetry based standards of absorbed dose to water," Technical Reports Series 398, Vienna, Austria, 2000.

[16] AERB Safety Guide, "Codes, standards and guides to be prepared by the regulatory body for nuclear and radiation facilities, AERB/RSD/RT/COM," Atomic Energy Regulatory Board, Mumbai, India, 2001.

[17] Portal Imaging and Portal Dosimetry Reference Guide, Varian Medical Systems, Palo Alto, Calif, USA, 2008.

[18] T. Depuydt, A. van Esch, and D. P. Huyskens, "A quantitative evaluation of IMRT dose distributions: refinement and clinical assessment of the gamma evaluation," Radiotherapy and Oncology, vol. 62, no. 3, pp. 309-319, 2002.

[19] R. Brun and F. Rademakers, "ROOT_an object oriented data analysis framework," Nuclear Instruments and Methods in Physics Research A, vol. 389, no. 1-2, pp. 81-86, 1997, Proceedings of the AIHENP '96 Workshop, Lausanne, Switzerland, September 1996. 


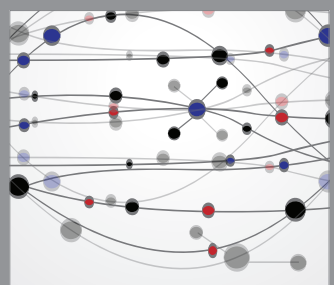

The Scientific World Journal
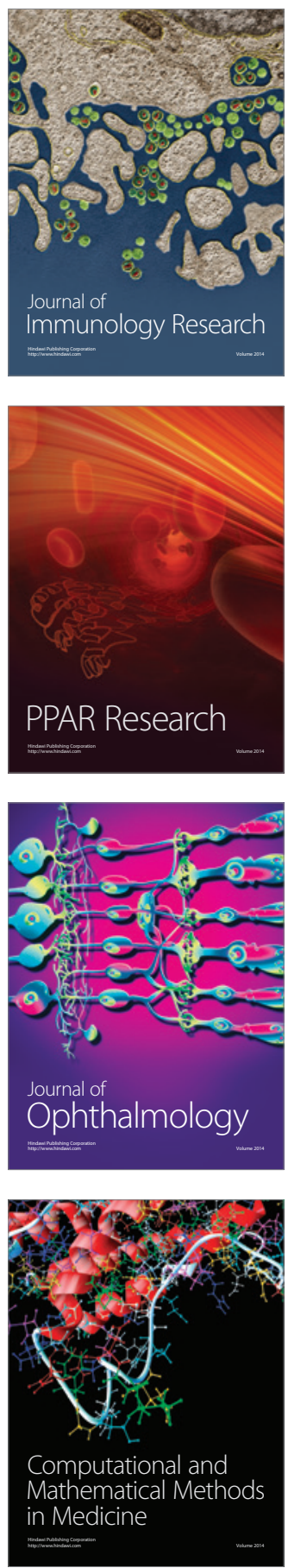

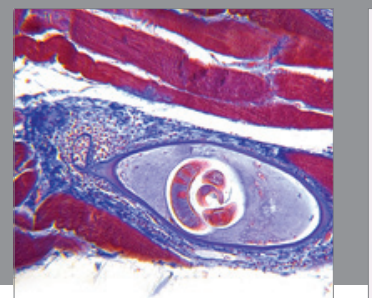

Gastroenterology

Research and Practice
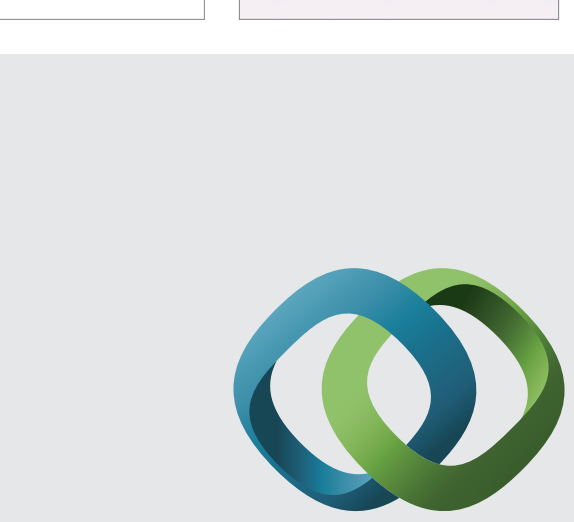

\section{Hindawi}

Submit your manuscripts at

http://www.hindawi.com
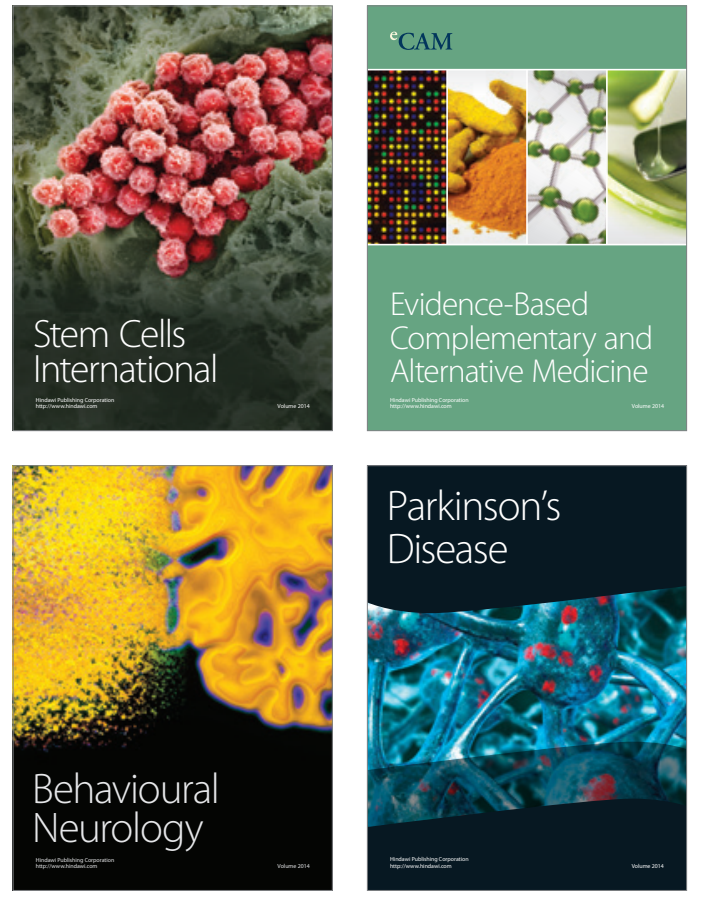
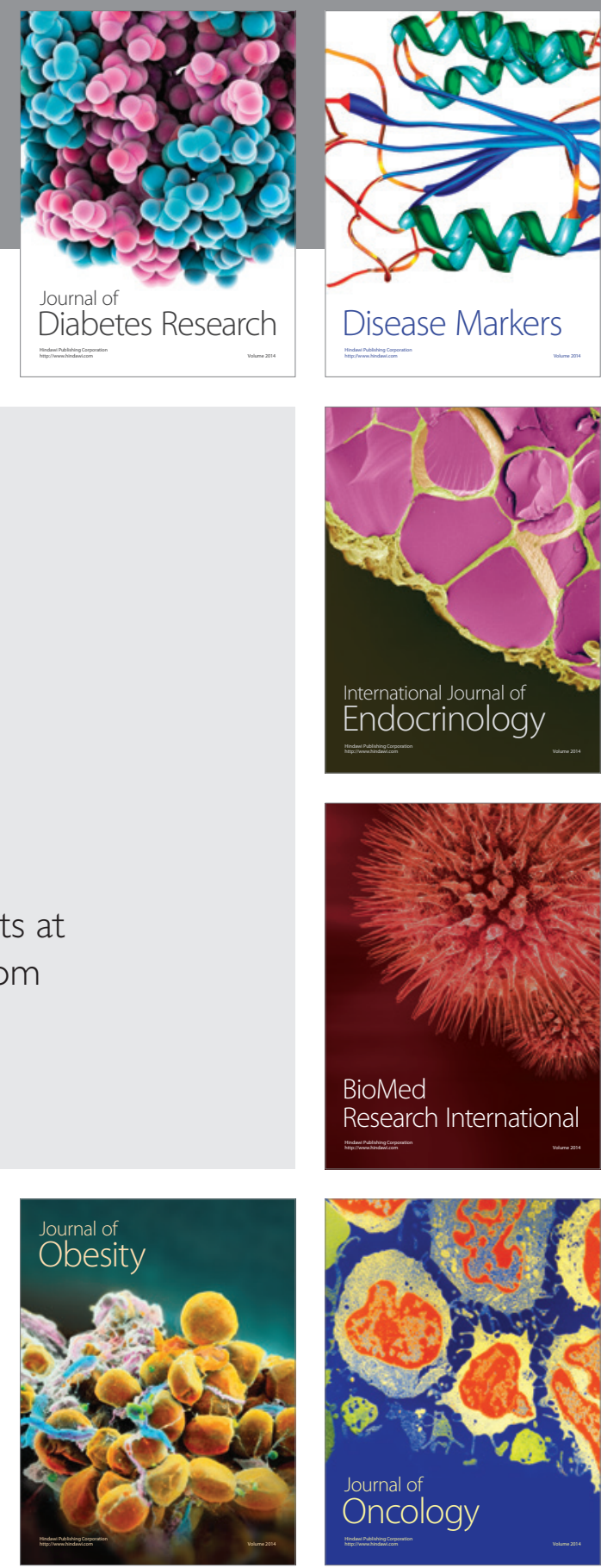

Disease Markers
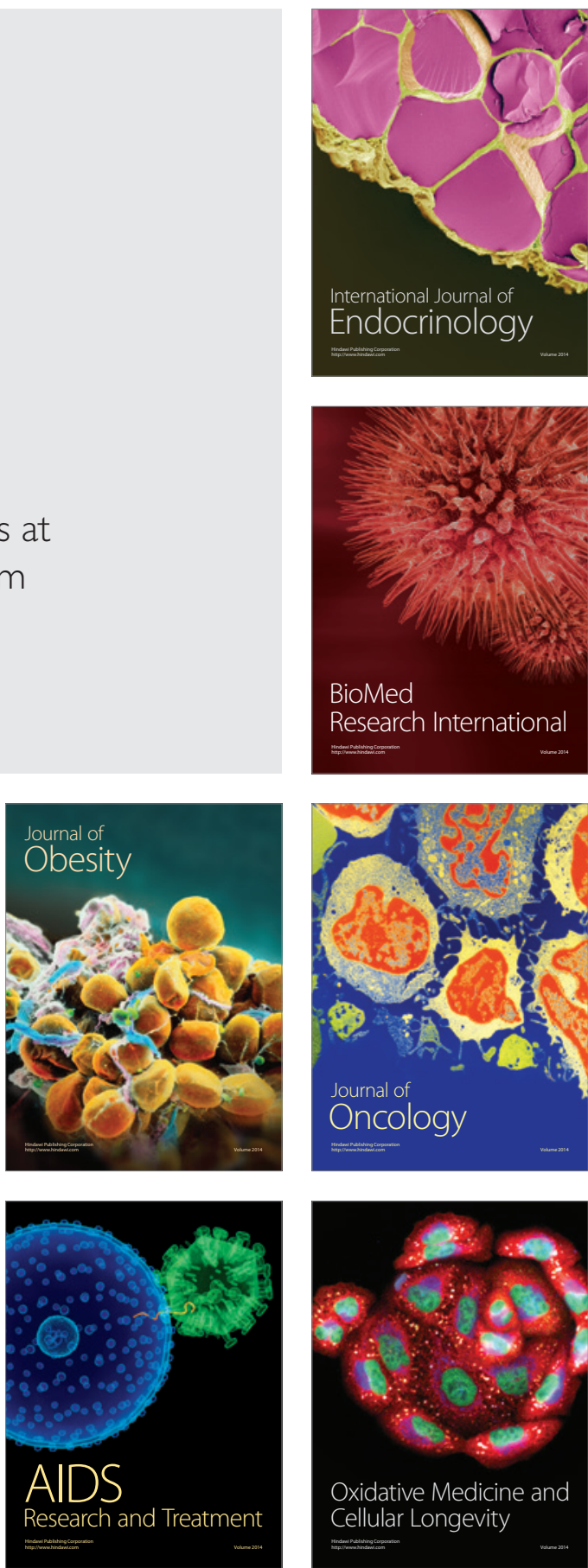